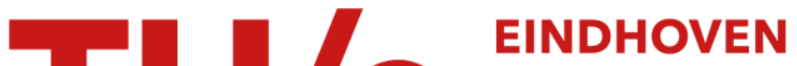 UNIVERSITY OF TECHNOLOGY
}

\section{Analog implementation of a robust control strategy for mechanical systems}

\section{Citation for published version (APA):}

Alvarez, J., Rosas, D., \& Pena Ramirez, J. (2009). Analog implementation of a robust control strategy for mechanical systems. IEEE Transactions on Industrial Electronics, 56(9), 3377-3385.

https://doi.org/10.1109/TIE.2009.2020706

DOI:

10.1109/TIE.2009.2020706

Document status and date:

Published: 01/01/2009

\section{Document Version:}

Publisher's PDF, also known as Version of Record (includes final page, issue and volume numbers)

\section{Please check the document version of this publication:}

- A submitted manuscript is the version of the article upon submission and before peer-review. There can be important differences between the submitted version and the official published version of record. People interested in the research are advised to contact the author for the final version of the publication, or visit the $\mathrm{DOI}$ to the publisher's website.

- The final author version and the galley proof are versions of the publication after peer review.

- The final published version features the final layout of the paper including the volume, issue and page numbers.

Link to publication

\section{General rights}

Copyright and moral rights for the publications made accessible in the public portal are retained by the authors and/or other copyright owners and it is a condition of accessing publications that users recognise and abide by the legal requirements associated with these rights.

- Users may download and print one copy of any publication from the public portal for the purpose of private study or research.

- You may not further distribute the material or use it for any profit-making activity or commercial gain

- You may freely distribute the URL identifying the publication in the public portal.

If the publication is distributed under the terms of Article 25fa of the Dutch Copyright Act, indicated by the "Taverne" license above, please follow below link for the End User Agreement:

www.tue.nl/taverne

Take down policy

If you believe that this document breaches copyright please contact us at:

openaccess@tue.nl

providing details and we will investigate your claim. 


\title{
Analog Implementation of a Robust Control Strategy for Mechanical Systems
}

\author{
Joaquin Alvarez, Member, IEEE, David Rosas, and Jonatan Peña
}

\begin{abstract}
An analog implementation of a control structure with disturbance identification for mechanical systems is presented. This control structure is based on a discontinuous observer that estimates the state and the disturbances in the plant, improving the robustness of the closed loop system which also displays a control input free from chattering. The control structure, built with operational amplifiers, is simple, showing good performance for stabilization, regulation, and tracking objectives. The performance of the circuit is compared with a digital implementation based on a real-time data acquisition card to control a simple pendulum. In addition, the circuit performance is illustrated on an industrial 2-DOF robot.
\end{abstract}

Index Terms-Free chattering, nonlinear observer, robustness, sliding modes.

\section{INTRODUCTION}

$\mathbf{C}$ ONTROL of mechanical systems based on a Lagrangian representation has been an important engineering problem for a long time. Most of the control algorithms proposed for these systems rely on models where practical situations like parameter uncertainty, external disturbances, or friction force terms are not considered. In addition, a complete availability of the state vector is often assumed. However, the position vector is usually the only available measurement; in consequence, the velocity vector must be calculated indirectly, yielding an inaccurate velocity.

Some works based on robust control techniques have been proposed to cope with parameter uncertainty and external disturbances, particularly $H_{\infty}$ and discontinuous control (see, for example, [1], [2], and references therein). A control procedure with good robustness properties for some kind of disturbances is the sliding mode technique. This control algorithm displays good performance for regulation and tracking objectives in mechanical systems [3]. Unfortunately, when the state reaches

Manuscript received October 6, 2008; revised February 14, 2009. First published April 21, 2009; current version published August 12, 2009. This work was supported in part by the CONACyT under Grant 48907 and in part by PROMEP under Grant 9848 .

J. Alvarez is with the Department of Electronics and Telecommunications, Scientific Research and Higher Education Center of Ensenada, 22860 Ensenada, BC, Mexico (e-mail: jqalvar@ cicese.mx).

D. Rosas is with the Engineering Faculty, Autonomous University of Baja California, 21280 Mexicali, BC, Mexico (e-mail: drosas@uabc.mx).

J. Peña is with the Dynamics and Control Group, Department of Mechanical Engineering, Eindhoven University of Technology, 5600 Eindhoven, The Netherlands.

Color versions of one or more of the figures in this paper are available online at http://ieeexplore.ieee.org.

Digital Object Identifier 10.1109/TIE.2009.2020706 the discontinuity surface the control input usually produces the chattering phenomenon, due to system deficiencies such as delays and hysteresis. In practice, chattering produces heat losses in power electronic circuits and wear of pieces as gears, transmissions, and bearings in mechanical systems [2].

Due to the attractive robustness characteristics of sliding mode control, several solutions to alleviate or even eliminate chattering have been developed for some kinds of systems [4], [5]. In these references, it is assumed that full state vector is measured. However, as it is well known, in practice, it is common to deal with systems where only some states are available due to, among other reasons, technological or economical limitations.

To solve the observation problem of uncertain systems several approaches have been developed. Sliding mode control has been also applied successfully to design observers [2], [6]-[8]. The sliding mode observers open the possibility to use the equivalent output injection to identify disturbances and uncertain parameters; this approach is considered in [6] and [8]. In addition, control techniques that use states estimated from sliding mode observers have been developed for linear systems [9] and for the nonlinear case [10].

Two interesting proposals to solve the chattering problem are [11] and [12]. In [11], a controller for mechanical systems based on sliding modes, where an approximation of the equivalent control instead of the signum function is used in the control law, is presented. This proposal needs a nominal model of the mechanism and a complete access to the state vector. Moreover, because the equivalent control is obtained in numerical form, the control algorithm needs to be implemented in a sufficiently fast processor to guarantee the stability of the closed loop system. In [12], a technique that automatically fits the width of the boundary layer in a sliding mode control system is proposed. This technique is based on fuzzy logic, with the objective to eliminate the chattering problem and maintain the robustness of the closed loop system. This technique is implemented in a dSpace 1102 real-time system, and applied to control a selective compliant assembly robot arm (SCARA) robot, obtaining steady-state errors of about $3.0 \times 10^{-5} \mathrm{rad}$.

In general, the control techniques mentioned before yield good control performance. However, the mathematical operations needed to calculate the control signal are rather complex, possibly due to the compensation of gravitational, centrifugal, or Coriolis terms, the solution of Hamilton-Jacobi-Isaacs equations, or the evaluation of the equivalent control. In addition, if an observer is included in the control system, the overall controller becomes dynamical, and the sampling time becomes 


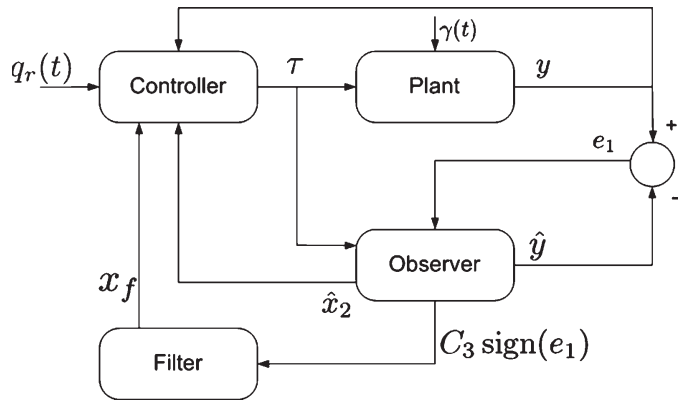

Fig. 1. Block diagram of the control structure with identification of disturbances for mechanical systems.

an important issue for the practical implementation. It is thus necessary to use a dedicated real-time system for the accomplishment of the control tasks. This increases the cost of the control system, which will need a fast processor if many highly complex numerical operations should be performed.

As an alternative to fast processors, the analog circuit technology can be considered. Some approaches to analogical implementation of control systems have been proposed [13], [14]. An important issue is that the circuit is particular to the controlled plant. In [15], software for the automatic design of analog circuits to control linear systems, based on evolutionary synthesis of analog circuits, is presented.

In this paper, we evaluate an analog implementation of a robust control structure to control a general class of mechanical systems. It solves the regulation and tracking objectives for this kind of system and is robust with respect to parameter uncertainties and external disturbances. This control structure needs only the position vector and guarantees asymptotic convergence to the reference, with a control input theoretically free from chattering. It includes a discontinuous observer that estimates the state vector and the plant disturbances, using the equivalent output injection concept. This estimated perturbation is included in the controller to compensate the actual disturbances affecting the plant. The robustness of the control structure permits us to design a simple electronic circuit, based on operational amplifiers, where complex mathematical operations are not necessary. These circuits allow us to decrease the load of the computer where the control task is programmed. We present experimental results of the performance of the circuit and a comparison with a digital implementation of the control structure.

This paper is organized as follows. Section II includes a description of the control structure. In Section III, we present a closed-loop stability analysis. The design with electronic circuits of each block composing the control structure is presented in Section IV. In Section V, the analysis of the control circuit performance is presented. Finally, a discussion of the results and the conclusions are included in Section VI.

\section{Description of Control Structure With IDENTIFICATION OF DISTURBANCES}

A block diagram of the control structure is shown in Fig. 1. The components are the plant, which is a mechanical system, a discontinuous observer, a low-pass filter, and the controller.

\section{A. Plant}

Consider an $n$ DOF mechanical system described by

$$
M(q) \ddot{q}+C(q, \dot{q}) \dot{q}+G(q)+\varphi(\ddot{q}, \dot{q}, q) \theta+\gamma(t)=\tau
$$

where $q, \dot{q}$, and $\ddot{q}$ are the position, the velocity, and the acceleration vectors, respectively, with dimension $n, M(q)$ is the inertia matrix, $C(q, \dot{q})$ is the Coriolis and centrifugal force matrix, $G(q)$ is the gravitational force vector, $\varphi(\ddot{q}, \dot{q}, q) \theta$ contains all the uncertain terms due to the parameter variations vector $\theta$. $\gamma(t) \in \Re^{n}$ is a bounded vector of external disturbances, and $\tau \in \Re^{n}$ is the control input. All matrices and vectors are defined with suitable dimensions. In this analysis, it is considered that only the position vector $q$ is available.

Defining the state variables as $x_{1}=q$ and $x_{2}=\dot{q}$, the state space representation of (1) is

$$
\begin{aligned}
\dot{x}_{1} & =x_{2} \\
\dot{x}_{2} & =f(x)+g\left(x_{1}\right)+\xi(\cdot)+M^{-1}\left(x_{1}\right) \tau \\
y & =x_{1}
\end{aligned}
$$

where

$$
\begin{aligned}
f(x) & =-M^{-1}\left(x_{1}\right) C\left(x_{2}, x_{1}\right) x_{2} \\
g\left(x_{1}\right) & =-M^{-1}\left(x_{1}\right) G\left(x_{1}\right) \\
\xi(\cdot) & =-M^{-1}\left(x_{1}\right)\left(\varphi\left(\dot{x}_{2}, x_{2}, x_{1}\right) \theta+\gamma(t)\right) .
\end{aligned}
$$

It can be seen that the effects of parameter variations and external disturbances are lumped in a single term and considered as a perturbation, called $\xi(\cdot)$. Note that the right-hand side of (2) is Lipschitz in $x_{1}$ and $x_{2}$, and piecewise continuous in $t$.

\section{B. Discontinuous Observer}

A discontinuous observer is included in the control structure to estimate the whole state vector of the plant. This observer is a simplified version of the observer presented in [6]. It guarantees exponential convergence to the state of the plant in spite of uncertainties and disturbances. Note that the perturbations in system (2) and (3) satisfy the observer matching conditions given in [16], which permits the design of an observer.

This observer plays a central role in the performance of the control structure. It estimates the state vector and, with the aid of a low-pass filter, it identifies also the perturbation term. The observer is given by

$$
\begin{aligned}
{\left[\begin{array}{c}
\dot{\hat{x}}_{1} \\
\dot{\hat{x}}_{2}
\end{array}\right] } & =\left[\begin{array}{c}
\hat{x}_{2} \\
M^{-1}\left(x_{1}\right) \tau
\end{array}\right]+H(y-\hat{y}) \\
\hat{y} & =\hat{x}_{1}
\end{aligned}
$$

where $\hat{x}_{1} \in \Re^{n}$ and $\hat{x}_{2} \in \Re^{n}$ are the states of the observer and the vector $H(y-\hat{y})$ has the form

$$
H(y-\hat{y})=\left[\begin{array}{c}
C_{1}(y-\hat{y}) \\
C_{2}(y-\hat{y})+C_{3} \operatorname{sign}(y-\hat{y})
\end{array}\right] .
$$


$C_{i}, i=1,2,3$ is a diagonal positive-definite matrix given by

$$
C_{i}=\operatorname{diag}\left\{c_{i, 1}, c_{i, 2}, \ldots, c_{i, n}\right\}, \quad \text { for } i=1,2,3
$$

and the signum vector function $\operatorname{sign}(y-\hat{y})$ is defined as

$$
\operatorname{sign}(y-\hat{y})=\left[\operatorname{sign}\left(y_{1}-\hat{y}_{1}\right), \ldots, \operatorname{sign}\left(y_{n}-\hat{y}_{n}\right)\right]^{\mathrm{T}} .
$$

Defining error variables as $e_{1}=x_{1}-\hat{x}_{1}, e_{2}=x_{2}-\hat{x}_{2}$, the error dynamics are described by

$$
\left[\begin{array}{c}
\dot{e}_{1} \\
\dot{e}_{2}
\end{array}\right]=\left[\begin{array}{c}
e_{2}-C_{1} e_{1} \\
f(x)+g\left(x_{1}\right)+\xi(\cdot)-C_{2} e_{1}-C_{3} \operatorname{sign}\left(e_{1}\right)
\end{array}\right] .
$$

Now, a new perturbation term is defined as

$$
\Psi(\cdot)=f(x)+g\left(x_{1}\right)+\xi(\cdot) .
$$

Note that, provided $q, \dot{q}, \ddot{q}$, and the external disturbance $\gamma(t)$ were bounded, then $\Psi(\cdot)$ is also bounded; that is

$$
\|\Psi(\cdot)\| \leq \rho_{1} \quad \forall t \geq 0
$$

where $\rho_{1}$ is a positive constant.

Theorem 1: If (8) is satisfied, then there exist matrices $C_{1}$, $C_{2}$, and $C_{3}$ such that the origin of the error space is an exponentially stable equilibrium point. Therefore, $\lim _{t \rightarrow \infty} \hat{x}(t)=x(t)$.

Proof: The proof of this theorem can be found in [6].

In [6], it is also shown that the equivalent output injection $u_{\text {eq }}$ is given by

$$
u_{\mathrm{eq}}=f(x)+g\left(x_{1}\right)+\xi(\cdot)=\Psi(\cdot) .
$$

This means that $u_{\mathrm{eq}}$ corresponds to the perturbation term that is formed by uncertainties, external disturbances and the terms $f(x)$ and $g\left(x_{1}\right)$. The last result opens the possibility to obtain the equivalent output injection $u_{\text {eq }}$ to include it into the controller to compensate the perturbation present in the plant.

\section{Filter}

According to [17], the equivalent output injection $u_{\text {eq }}$ coincides with the slow component of the discontinuous term in (6) when the state slides on the discontinuity surface. Thus, it can be recovered using a low-pass filter whose time constant is small enough as compared with the slow component, yet sufficiently large to filter out the high-rate components. Then, we propose to use a set of $n$ second-order low-pass Butterworth filter to estimate the term $u_{\text {eq }}$.

The following normalized transfer function is used for each filter:

$$
\frac{Y_{i}(s)}{U_{i}(s)}=\frac{w_{\mathrm{c}_{i}}^{2}}{s^{2}+1.4142 w_{\mathrm{c}_{i}} s+w_{\mathrm{c}_{i}}^{2}}, \quad i=1, \ldots, n
$$

where $w_{\mathrm{c}_{i}}$ is the cutoff frequency of each filter. Here, the filter input is the discontinuous term of the observer $c_{3, i} \operatorname{sign}\left(y_{i}-\right.$ $\left.\hat{y}_{i}\right)$. Denoting the output of the set of filters as $x_{\mathrm{f}} \in \Re^{n}$ and choosing a set of constants $w_{\mathrm{c}_{i}}$ that minimizes the phase delay, it is possible to assume that

$$
\lim _{t \rightarrow \infty} x_{\mathrm{f}}=\tilde{\Psi}(\cdot) \approx \Psi(\cdot)
$$

where $\|\tilde{\Psi}(\cdot)-\Psi(\cdot)\| \leq \rho_{0}$ for $\rho_{0} \ll \rho_{1}$.

\section{Controller}

The controller can be given by any control algorithm to which the $x_{\mathrm{f}}$ term is added to compensate the disturbances acting on the plant. For example, in this paper a proportionalderivative (PD) controller with inertia matrix compensation is used. The control objective is to track a smooth reference signal $q_{\mathrm{r}}(t) \in \Re^{n}$. Hence, the controller is given by the expression

$$
\begin{aligned}
\tau=M\left(x_{1}\right)\left[-x_{\mathrm{f}}-\right. & K_{\mathrm{p}}\left(x_{1}-q_{\mathrm{r}}(t)\right) \\
& \left.-K_{\mathrm{v}}\left(\hat{x}_{2}-\dot{q}_{\mathrm{r}}(t)\right)\right]+M\left(x_{1}\right) \ddot{q}_{\mathrm{r}}(t)
\end{aligned}
$$

where $K_{\mathrm{p}}$ and $K_{\mathrm{v}}$ are positive-definite diagonal matrices.

To compensate the small difference between $x_{\mathrm{f}}$ and $u_{\text {eq }}$, a second loop can be included (for example, $H_{\infty}$ or sliding mode controllers). However, this introduces an additional complexity in the controller implementation.

\section{Stability Analysis of Control Structure}

The dynamics of the error variables $e_{1}=x_{1}-\hat{x}_{1}, e_{2}=$ $x_{2}-\hat{x}_{2}$ takes the form (6), and applying the Theorem 1, it is possible to conclude that the observer converges exponentially to the state of the plant.

Now, defining the tracking error between the plant output and the reference signal $z_{1}=x_{1}-q_{\mathrm{r}}$, and its time derivatives $z_{2}=$ $x_{2}-\dot{q}_{\mathrm{r}}$, the dynamics of these error varables are given by

$$
\begin{aligned}
& \dot{z}_{1}=z_{2} \\
& \dot{z}_{2}=\varpi(\cdot)-K_{\mathrm{p}} z_{1}-K_{\mathrm{v}} z_{2}
\end{aligned}
$$

where $\varpi=\Psi(\cdot)-x_{\mathrm{f}}-K_{\mathrm{v}} e_{2}$ is defined as a perturbation term with the bound

$$
\left\|\Psi(\cdot)-x_{\mathrm{f}}-K_{\mathrm{v}} e_{2}\right\| \leq \alpha e^{-\beta t}+\rho_{0} .
$$

Taking into account that the nominal system, defined with $\varpi(\cdot)=0$ in (12), has the origin as an exponentially stable equilibrium point, and considering the results on stability of perturbed systems presented in [18], we can guarantee that

$$
\|z\| \leq \varepsilon \quad \forall t \geq T
$$

where $T$ is a finite time and $\varepsilon$ is a small constant.

For the ideal case, considering a limit procedure when the boundary layer width in the real control tends to zero and an ideal filter, we have

$$
\lim _{t \rightarrow \infty} x_{\mathrm{f}}(t)=\Psi(\cdot)
$$

in consequence

$$
\lim _{t \rightarrow \infty}\|\varpi\|=\lim _{t \rightarrow \infty}\left\|\Psi(\cdot)-x_{\mathrm{f}}-K_{\mathrm{v}} e_{2}\right\|=0 .
$$




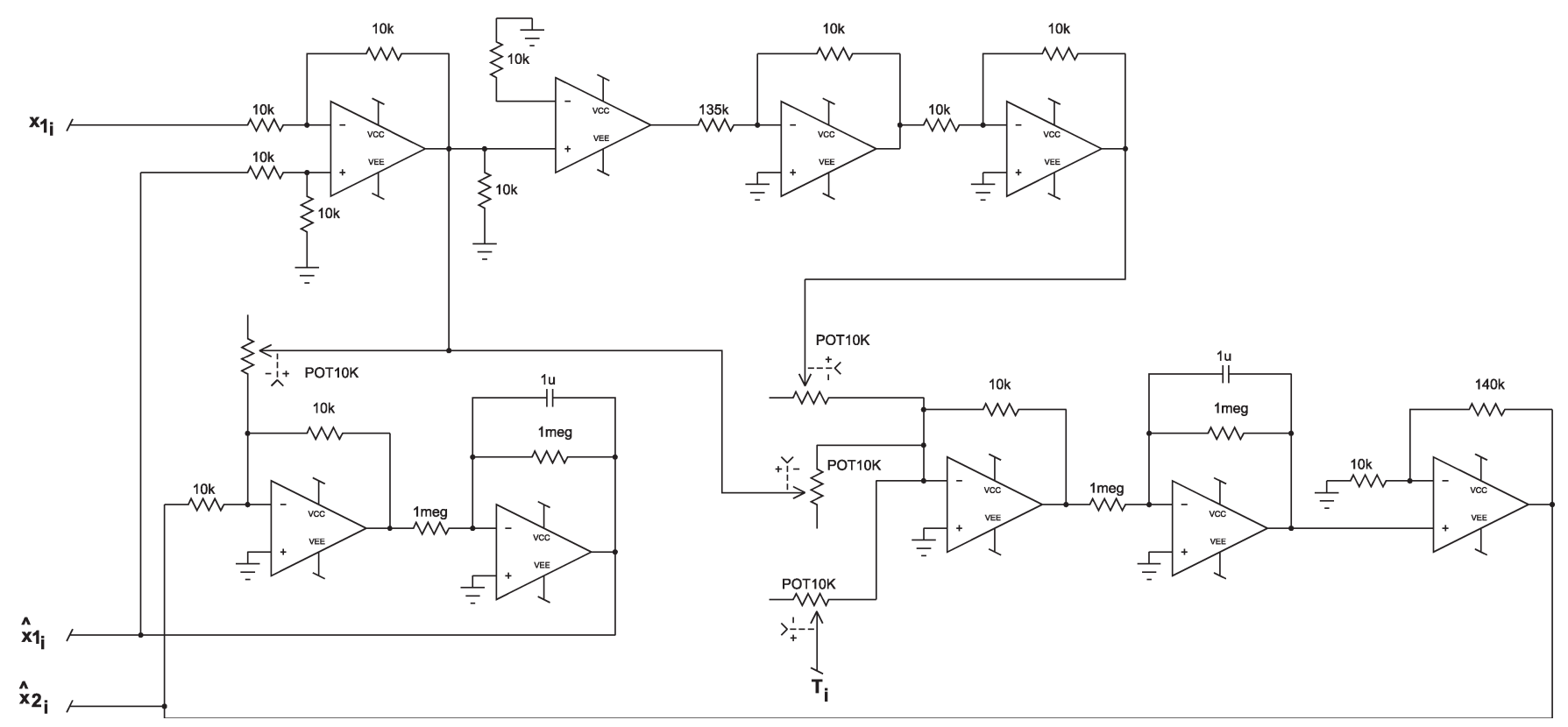

Fig. 2. Electronic circuit of the observer.

The term $\varpi(\cdot)$ can be seen as a vanishing perturbation with exponential decreasing rate. Hence, it is possible to find values for $K_{\mathrm{p}}$ and $K_{\mathrm{v}}$ such that the origin of the system (12) will be an asymptotically stable equilibrium point [18].

\section{Design of Analog Circuit of CONTROL STRUCTURE}

To design an electronic circuit for this structure which can be used for different mechanisms modeled by (1), it is convenient to express the inertia matrix $M(q)$ as

$$
M(q)=M_{0}+\Delta M(q)
$$

where $M_{0}=\operatorname{diag}\left\{m_{1}, \ldots, m_{n}\right\}$ is a nominal diagonal constant matrix and $\Delta M(q)=M(q)-M_{0}$ will be considered as a perturbation term that will be compensated by the controller.

Then, the control structure can be seen as set of $n 1$-DOFsubsystems with a plant given by

$$
\begin{aligned}
\dot{x}_{1_{i}} & =x_{2_{i}} \\
\dot{x}_{2_{i}} & =f_{i}(x)+g_{i}\left(x_{1}\right)+\xi_{i}(\cdot)+m_{i}^{-1} \tau_{i} \\
y_{i} & =x_{1 i}
\end{aligned}
$$

an observer with the form

$$
\begin{aligned}
{\left[\begin{array}{c}
\dot{\hat{x}}_{1_{i}} \\
\dot{\hat{x}}_{2_{i}}
\end{array}\right] } & =\left[\begin{array}{c}
\hat{x}_{2_{i}} \\
m_{i}^{-1} \tau_{i}
\end{array}\right]+\left[\begin{array}{c}
c_{1_{i}}\left(y_{i}-\hat{y}_{i}\right) \\
c_{2_{i}}\left(y_{i}-\hat{y}_{i}\right)+c_{3_{i}} \operatorname{sign}\left(y_{i}-\hat{y}_{i}\right)
\end{array}\right] \\
\hat{y}_{i} & =\hat{x}_{1_{i}}
\end{aligned}
$$

a filter given by (9), and a controller given by

$$
\begin{aligned}
\tau_{i}=m_{i}\left[-x_{\mathrm{f}_{i}}-k_{\mathrm{p}_{i}}(\right. & \left.x_{1_{i}}-q_{\mathrm{r}_{i}}(t)\right) \\
& \left.-k_{\mathrm{v}_{i}}\left(\hat{x}_{2_{i}}-\dot{q}_{\mathrm{r}_{i}}(t)\right)\right]+m_{i} \ddot{q}_{\mathrm{r}_{i}}(t)
\end{aligned}
$$

for $i=1, \ldots, n$. Equations (9) and (15)-(17) form a module to control each joint of system (2). In the sequel, we describe the design of the components of this module.

\section{A. Design of Observer Circuit}

The observer has been scaled by a factor of 15 to keep the magnitude of the signals into suitable levels. This scaling factor was determined through simulations. This scaling is performed only on signal $\hat{x}_{2, i}$ because it is considered that signal $\hat{x}_{1, i}$ does not attain values greater than $2 \pi \mathrm{V}$. However, the observer circuit may be scaled by another factor according to the magnitude of the signals.

By doing a change of variables, $\hat{x}_{1, i}=\bar{x}_{1, i}$ and $\hat{x}_{2, i}=$ $15 \bar{x}_{2, i}$, the observer dynamics are described by

$$
\begin{aligned}
\dot{\bar{x}}_{1, i}= & 15 \bar{x}_{2, i}+c_{1, i}\left(x_{1, i}-\bar{x}_{1, i}\right) \\
\dot{\bar{x}}_{2, i}= & \frac{m_{i}^{-1}}{15} \tau+\frac{c_{2, i}}{15}\left(x_{1}-\bar{x}_{1}\right) \\
& +\frac{c_{3, i}}{15} \operatorname{sign}\left(x_{1, i}-\bar{x}_{1, i}\right) .
\end{aligned}
$$

The electronic circuit is shown in Fig. 2, whereas a first step the observation error $e_{1, i}$ is calculated. In the second step the error signum is obtained. Finally, the observer is built with two integrators and two adders. Pertinent considerations have been done in such a way that the outputs of the circuit are the observed states. The JFET-input operational amplifier TL084, manufactured by Texas Instruments, is employed in the circuit.

\section{B. Design of Low-Pass Filter Circuit}

There are two well-known architectures for analog implementation of active filters, Sallen-Key and Rauch architectures. 


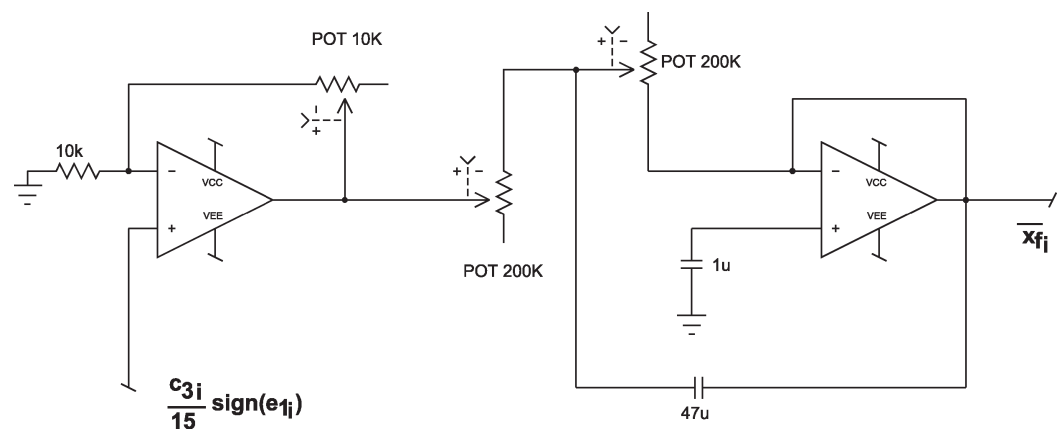

Fig. 3. Electronic circuit of the filter.

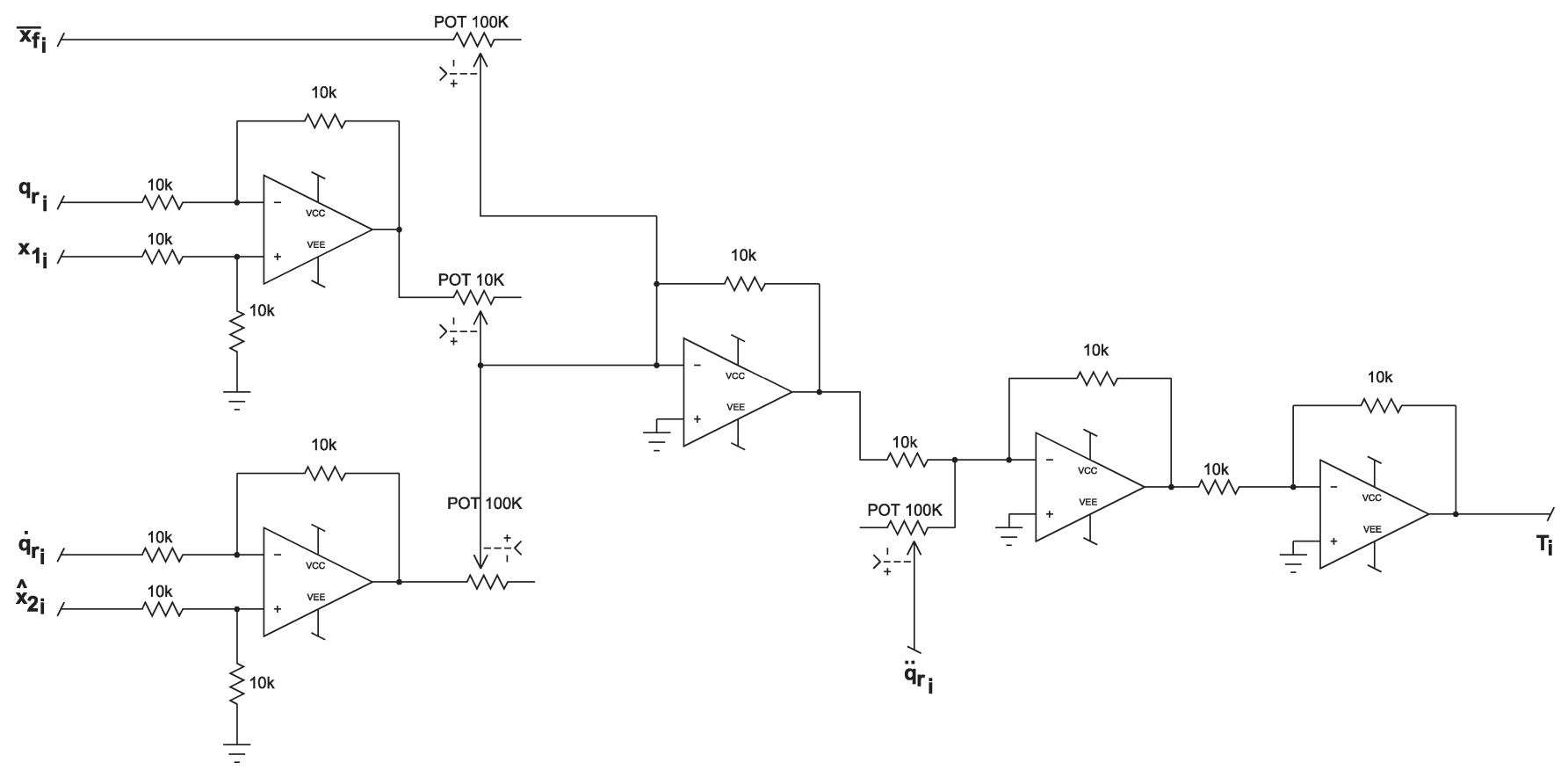

Fig. 4. Controller circuit.

The Sallen-Key architecture is used because it requires fewer components. The transfer function (9) can be written as

$$
\frac{Y_{i}(s)}{U_{i}(s)}=\frac{1}{w_{\mathrm{c}_{i}}^{2} R_{1, i} R_{2, i} C_{1, i} C_{2, i} s^{2}+w_{\mathrm{c}_{i}} C_{1, i}\left(R_{1, i}+R_{2, i}\right) s+1} .
$$

Because the filter input is scaled in a factor of 15 , the filter output is scaled by the same factor, denoting its output as $\bar{x}_{f_{i}}$. The filter circuit is shown in Fig. 3. In this circuit, the dual low-noise wide-bandwidth precision amplifier TL5580, manufactured by Texas Instruments, is employed in the implementation.

\section{Design of Controller Circuit}

The analog implementation of controller (11) is straightforward because it needs only algebraic operations. It is not necessary to scale the controller equation, it is only necessary to multiply by $M_{\mathrm{o}}$ each term before performing the addition.

The controller circuit is shown in Fig. 4. The JFET-input operational amplifier T1084, manufactured by Texas Instruments, is employed in the circuit.
The full circuit of the control structure with identification of disturbances is shown in Fig. 5. There is a new block in this circuit corresponding to a velocity filter. Its purpose is to reduce the high-frequency components that are present in the estimated velocity term $\hat{x}_{2}$. These high-frequency components are due to the discontinuous term present into the observer and to circuit imperfections. There is a selector to choose the signal which will be sent to the controller, $\hat{x}_{2}$ or $\hat{x}_{2}$ filtered. In some cases, when the coefficient $C_{3}$ is small enough, it is not necessary to filter $\hat{x}_{2}$. However, when this coefficient is large, it is convenient to use the filtered version because it reduces the high-frequency components in the control signal. The velocity filter is given by the equation

$$
\Gamma \dot{x}_{2 \mathrm{fil}}=-x_{2 \mathrm{fil}}+\hat{x}_{2}
$$

where $\Gamma=0.05$, and the filter output is $x_{2 \mathrm{fil}}$.

There is another selector that permits us to choose between the control signal $\tau$ or an auxiliary signal $\tau_{\text {aux }}$. The signal $\tau$ is selected for the closed-loop system, $\tau_{\text {aux }}$ when the system is not controlled. The signal $\tau_{\text {aux }}$ allows one to tune the observer when the system is in open loop. 


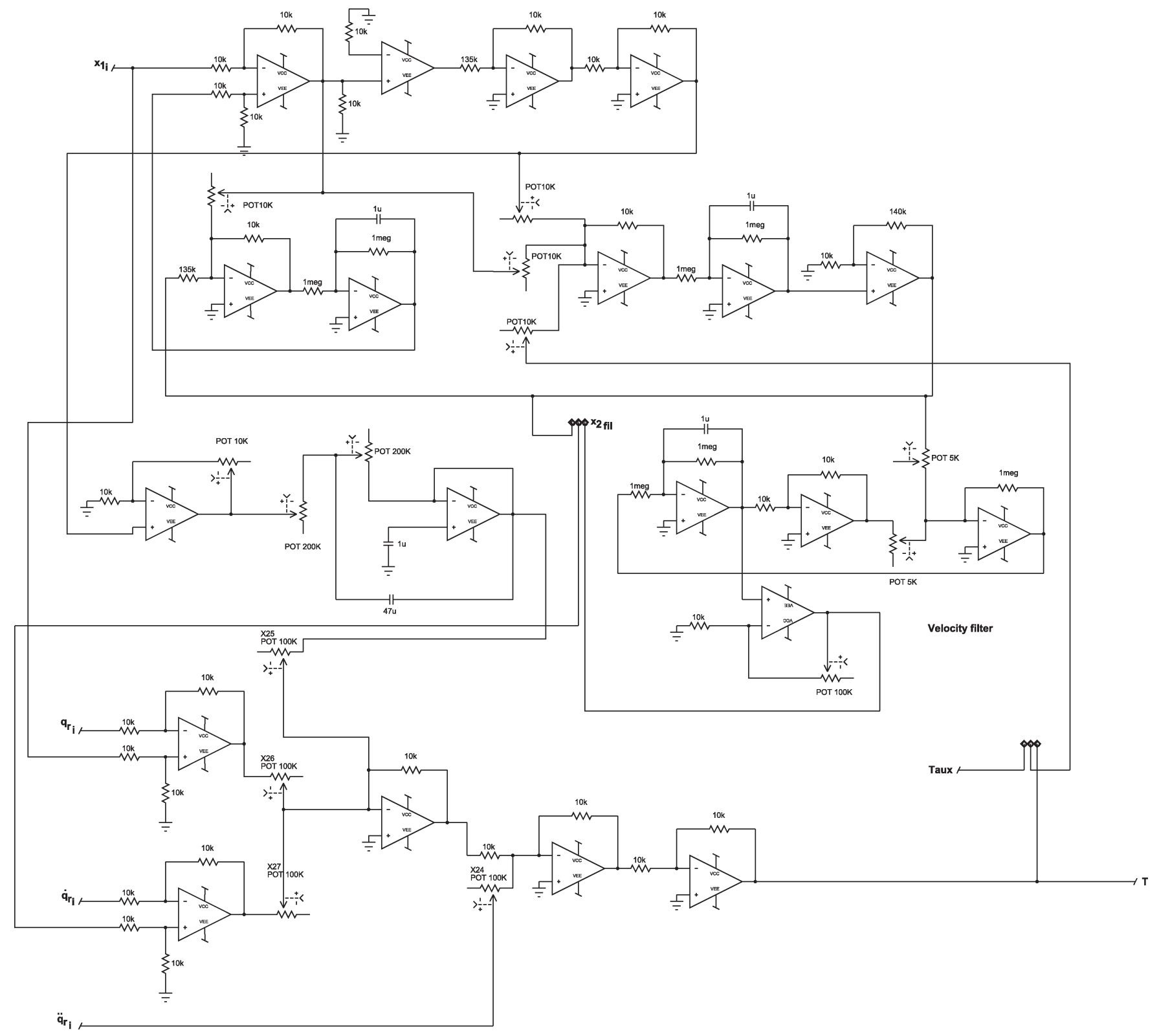

Fig. 5. Full circuit of the control structure with identification of disturbances.

There are several potentiometers which are labeled according to their corresponding gain. The tuning of the circuit is not difficult if one follow the theoretical development presented in previous sections.

\section{Analysis of Control Circuit Performance}

In this section, the performance of the electronic circuit is analyzed. This is carried on with the application to two mechanisms: a simple pendulum and an industrial robot.

\section{A. Application to Simple Pendulum of Analog and Digital Implementations}

The analog and digital implementation of the control structure is presented in this section to evaluate the performance of the analog circuit, the objective is to show that the performance of the circuit is similar to the performance of the computerbased control. In the digital implementation, the Simulink package of Matlab software, with solver ode2 (Heun), and a fixed step of $10^{-4} \mathrm{~s}$ were used, with a dSpace DS1104 system for real-time PC-based applications.

A mechanical simple pendulum of Mechatronics, Inc., is used as the plant, whose nominal model is given by

$$
\begin{aligned}
\dot{x}_{1} & =x_{2} \\
\dot{x}_{2} & =-a x_{2}-b \sin \left(x_{1}\right)+c \tau+\gamma(t) \\
y & =x_{1}
\end{aligned}
$$

where $a=\left(I+m l_{\mathrm{c}}^{2}\right)^{-1} \delta, \quad b=\left(I+m l_{\mathrm{c}}^{2}\right)^{-1}\left(-m g l_{\mathrm{c}}\right), \quad c=$ $\left(I+m l_{\mathrm{c}}^{2}\right)^{-1}$, with $m=1.6365 \mathrm{~kg}, l_{\mathrm{c}}=0.0762 \mathrm{~m}, g=$ $9.804 \mathrm{~m} / \mathrm{s}^{2}, I=0.0085 \mathrm{~kg} \cdot \mathrm{m}^{2}$, and $\delta=0.00053 \mathrm{~kg} \cdot \mathrm{m}^{2} / \mathrm{s}$. 


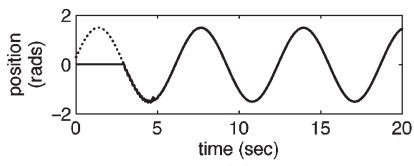

(a)

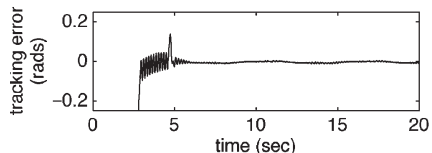

(b)

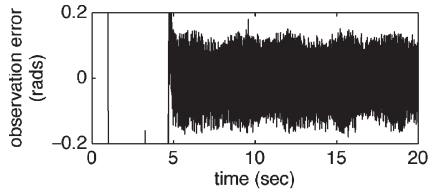

(c)

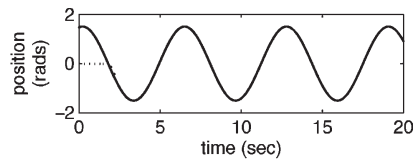

(d)

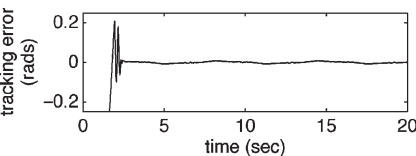

(e)

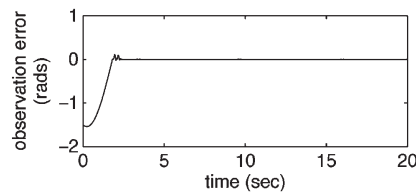

(f)
Fig. 6. Comparison between (left) analog and (right) digital implementation: Position, tracking error, and observation error for the simple pendulum.

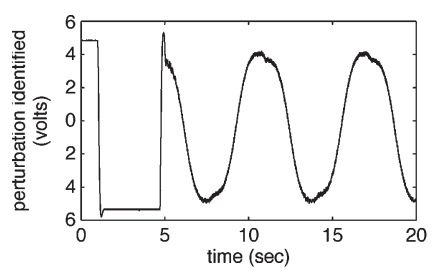

(a)

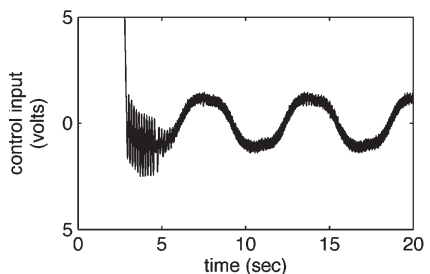

(b)

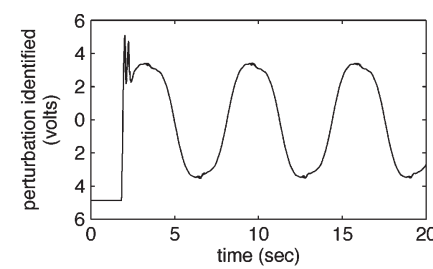

(c)

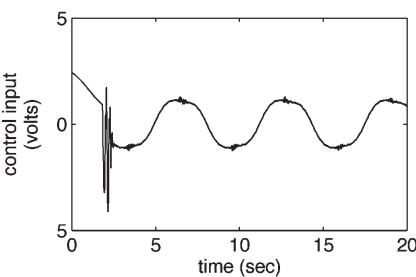

(d)
Fig. 7. Comparison between (left) analog and (right) digital: Perturbation identified and control input for the simple pendulum.

It is considered that $\gamma(t)$ represents a bounded external disturbance. The resistor values in the control circuit are $R_{C_{1}}=$ $400 \Omega, R_{C_{2}}=6 \mathrm{k} \Omega, R_{C_{3}}=1.667 \mathrm{k} \Omega, R_{K_{\mathrm{p}}}=1.388 \mathrm{k} \Omega$, and $R_{K_{\mathrm{v}}}=15.873 \mathrm{k} \Omega$, and the gains in the software are $C_{1}=25$, $C_{2}=25, C_{3}=90, K_{\mathrm{p}}=400$, and $K_{\mathrm{v}}=35$.

A Butterworth filter is designed with a cutoff frequency of $30 \mathrm{rad} / \mathrm{s}$. In all experiments $1 \mathrm{rad}$ is represented by $1 \mathrm{~V}$. It is important to note that in all experiments, due to an emergency stop button in the power drive, at the beginning the system is in open loop, some seconds later the loop is closed, it produces a big control signal at the beginning.

The comparison between analog and digital performance is shown in Figs. 6 and 7. In Fig. 6(a) and (d), it can be seen that the pendulum position reaches the reference signal in a short time, this means that the differences between the nominal model and the real model has been identified by the observer and the filter and compensated by the controller, this result cannot be reached with classical PD controller. The tracking errors, in steady state, keep levels lower than $1 \%$ that represents a deviation lower than $0.01 \mathrm{rad}$ [see Fig. 6(b) and (e)]. The observation errors, Fig. 6(c) and (f), also remain small which indicates the correct operation of the observer.

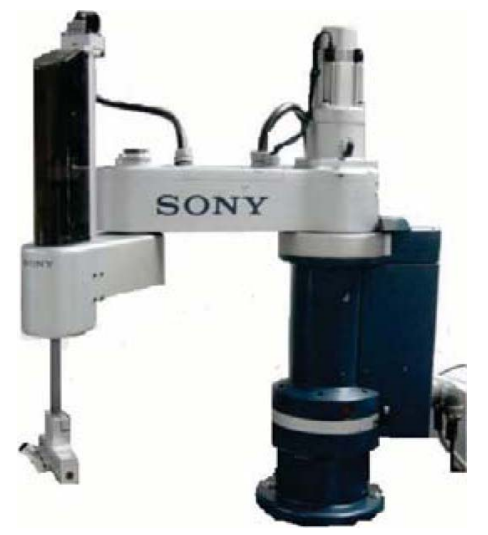

Fig. 8. SCARA robot controlled by the analog implementation of the control structure.

There is a difference between digital and analog implementation in the observation error. The signal shown in Fig. 6(c) is noisy due to the inherent noise present in electronic circuits. However, as it is shown in Fig. 6(a) and (b), the control objective is achieved with the same precision that for the digital implementation.

Fig. 7 shows the scaled estimated perturbation term [Fig. 7(a) and (c)]. To obtain the actual perturbation, it is necessary to multiply these signals by 15 . One can see that perturbation term, in the case of analog implementation, is larger than digital implementation. This is due to component tolerances of the circuit, which are considered as disturbances and included in this term.

Fig. 7 shows also the control inputs for the analog and digital cases. In the analog case, there are high-frequency components with very small amplitude. This is because the control signal has to compensate all the physical circuit imperfections. However, these components do not produce harmful effects to the mechanism.

\section{B. Application to Industrial Robot}

A SCARA industrial robot from SONY (see Fig. 8) is used to show the performance of the control circuit in an industrial equipment. In this experiment, there is an extreme situation; all parameters are unknown but the circuit used to control the simple pendulum will be used here with an adequate tuning. This experiment is restricted, due to some technical limitations, to $2 \mathrm{DOF}$ that form a horizontal double pendulum. The nominal inertia matrix with constant coefficients is given by

$$
M_{\mathrm{o}}^{-1}=\left[\begin{array}{cc}
55.549 & 0 \\
0 & 55.549
\end{array}\right]
$$

these constants were taken from the simple pendulum, here $x_{1,1}, x_{1,2}$ are the position and the velocity of the joint 1 and $x_{2,1}, x_{2,2}$ are the position and the velocity of the joint 2 . For each joint, a control circuit is used. The values of the resistors in both circuits are the following. For the joint 1 , $R_{C_{1}}=400 \Omega, R_{C_{2}}=6 \mathrm{k} \Omega, R_{C_{3}}=500 \Omega, R_{K_{\mathrm{p}}}=831 \Omega$, and $R_{K_{\mathrm{v}}}=1.462 \mathrm{k} \Omega$. For the joint $2, R_{C_{1}}=400 \Omega, R_{C_{2}}=6 \mathrm{k} \Omega$, $R_{C_{3}}=500 \Omega, R_{K_{\mathrm{p}}}=1418 \Omega$, and $R_{K_{\mathrm{v}}}=11.219 \mathrm{k} \Omega$. 


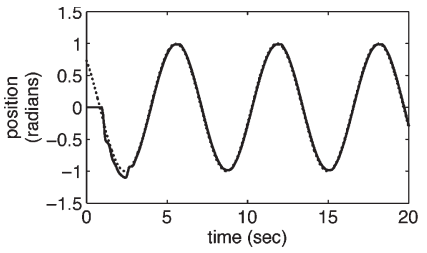

(a)

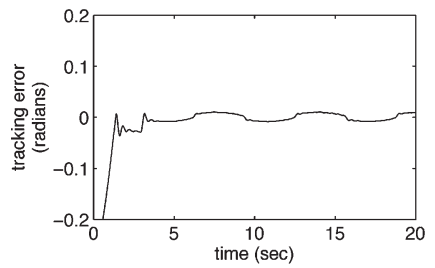

(c)

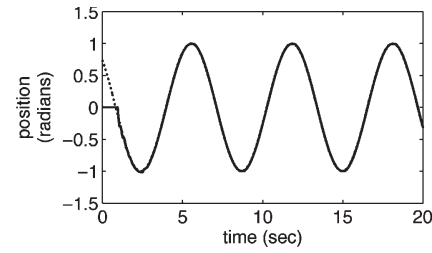

(b)

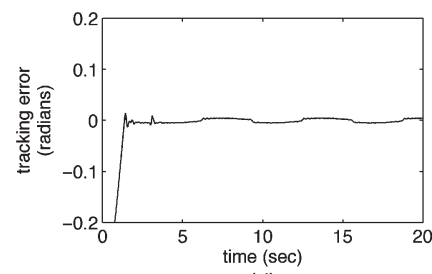

(d)
Fig. 9. Experimental results for the SCARA robot. Joint 1: (a) reference and joint position, (c) tracking error. Joint 2: (b) reference and joint position and (d) tracking error.

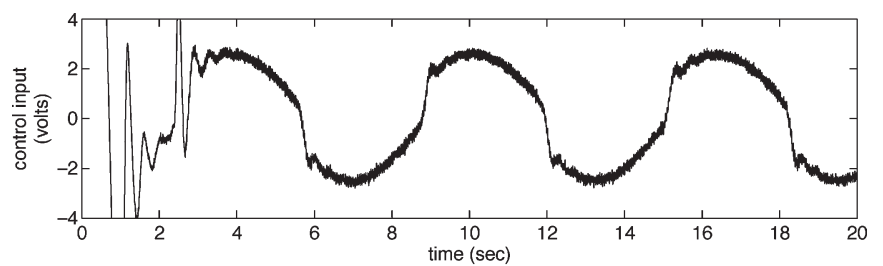

(a)

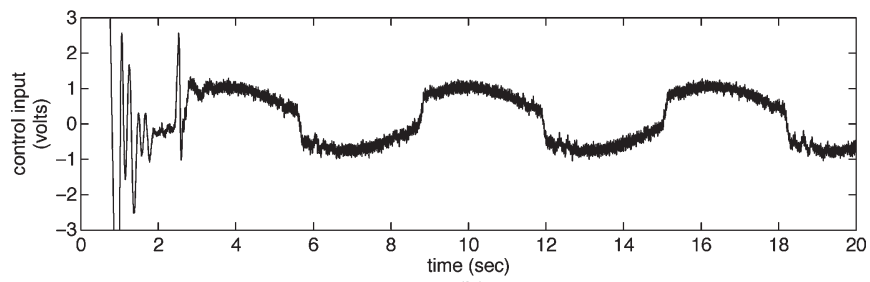

(b)

Fig. 10. Experimental results for the SCARA robot; the control signals. (a) Joint 1. (b) Joint 2.

The control objective is achieved as is shown in Fig. 9. In Fig. 9(a) and (b), it can be seen that the robot joint positions (solid lines) reach the reference signal (dotted lines) in a short time, having a good performance in steady state. In Fig. 9(c) and (d), the tracking errors are presented. These errors are small, lower than $1 \%$, which is satisfactory for many practical implementations.

Control input signals are shown in Fig. 10. For both control signals, the levels are into ranges not dangerous to operational amplifiers. Moreover, the high-frequency components that are present in the control input signals do not produce harmful effects as would happen with a controller based on sliding modes. The results obtained are acceptable in many industrial applications.

In a second experiment, a train of pulses is applied to each joint to simulate the typical operation for this kind of robots (see Fig. 11). Fig. 12 shows that the tracking errors in steady state are about $5 \times 10^{-4} \mathrm{rad}$. It is important to note that in this experiment, the maximum velocity in joint one is approximately $420 \%$ s and in the second joint is approximately $286^{\circ} \%$ s, these values are inside the typical operation range of this kind of the industrial robots (see, for example, [19]).
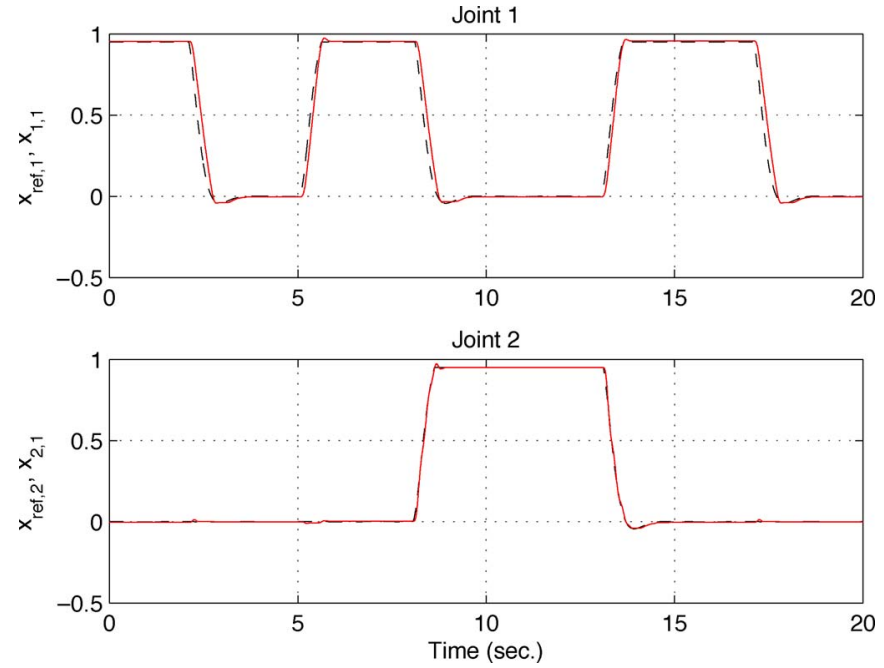

Fig. 11. Experimental results for the SCARA robot with a pulsed signal as reference.
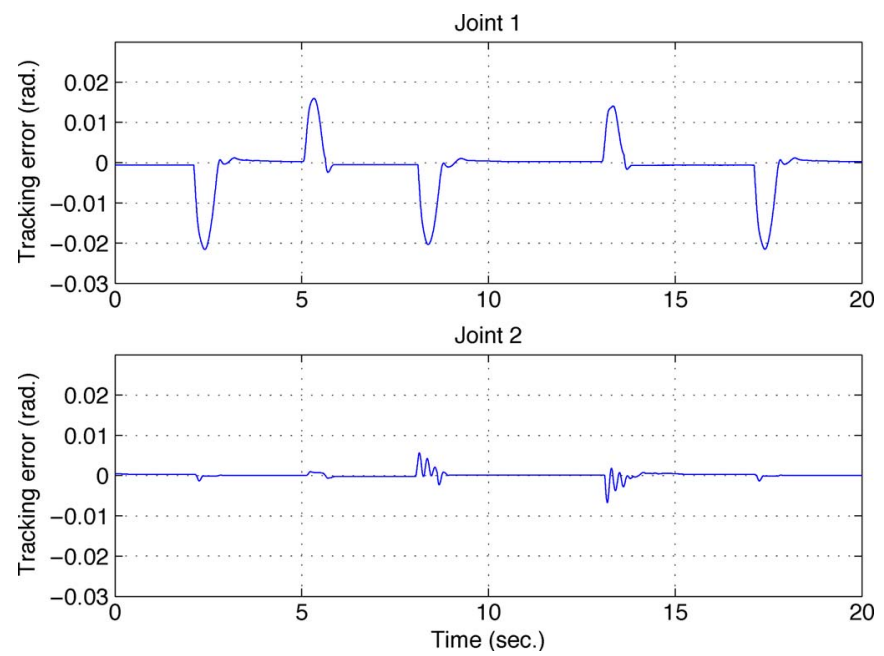

Fig. 12. Tracking errors for the SCARA robot with a pulsed signal as reference.

\section{DisCUSSION AND CONCLUSION}

This control structure presents interesting characteristics as robustness, identification of disturbances, modular implementation, and free chattering. It only needs the position of each joint and does not involve complex calculations. Therefore, it is easy to implement it with analog circuits. Its main limitation is that it can be applied only to Lagrangian systems.

The electronic circuit proposed in this paper presented a good performance for regulation and tracking of a SCARA robot. The steady-state regulation errors have magnitudes about $6.5 \times 10^{-4} \mathrm{rad}$ in the first joint and $8 \times 10^{-5} \mathrm{rad}$ in the second joint. These errors are very similar to those obtained in [12], where a dSpace 1102 system was used.

It is important to note that both systems, the simple pendulum and the SCARA robot, were controlled exactly with the same physical circuit, although their model and dimensions are very different. This shows the robustness and the flexibility of the circuit. 
The simplicity of the circuit may render possible implementation as an integrated circuit after a design optimization [15]. This will reduce the dimensions and the cost of the controller. It is also possible to implement this control structure in an FPGA, which allows an easy tuning and scaling of signals in the observer. See [20] and [21] for some examples of the implementation of a controller in an FPGA.

This control structure may be applied also to other systems with the Lagrangian structure, as microelectromechanical systems (MEMS). In [22], a numerical study of the application of a robust control to MEMS is presented, where external disturbances and parametric uncertainties are considered. In [23], a Lagrangian model is used to describe MEMS, and a comparison of the performance of three types of controller: sliding modes, high gain, and model-free control, is presented.

\section{REFERENCES}

[1] A. Isidori and A. Astolfi, "Disturbance attenuation and $\mathrm{H}_{\infty}$ control via measurement feedback in nonlinear systems," IEEE Trans. Autom. Control, vol. 37, no. 9, pp. 1283-1293, Sep. 1992.

[2] V. Utkin, J. Guldner, and J. Shi, Sliding Mode Control in Electromechanical Systems. London, U.K.: Taylor \& Francis, 1999.

[3] W. Yuzhuo and H. Flashner, "On sliding mode control of uncertain mechanical systems," in Proc. IEEE Aerosp. Conf., Aspen, CO, 1998, pp. 85-92.

[4] J.-X. Xu, Y.-J. Pan, and T.-H. Lee, "Sliding mode control with closedloop filtering architecture for a class of nonlinear systems," IEEE Trans. Circuits Syst., vol. 51, no. 4, pp. 168-173, Apr. 2004.

[5] V. Utkin and H. Chang, "Sliding mode control on electromechanical systems," Math. Probl. Eng., vol. 8, no. 4, pp. 451-473, 1999.

[6] D. Rosas, J. Alvarez, and L. Fridman, "Robust observation and identification of nDOF Lagrangian systems," Int. J. Robust Nonlinear Control, vol. 17, no. 9, pp. 842-861, Jun. 2006.

[7] R. Aguilar and R. Maya, "State estimation for nonlinear systems under model uncertainties: A class of sliding-mode observers," J. Process Control, vol. 15, no. 3, pp. 363-370, Apr. 2005.

[8] J. Davila, L. Fridman, and A. Poznyak, "Observation and identification of mechanical systems via second order sliding modes," Int. J. Control, vol. 79 , no. 10, pp. 1251-1262, 2006.

[9] H. Sira-Ramirez, S. Spurgeon, and K. Sara, "Robust sliding mode control using measured outputs," J. Math. Syst., Estimation Control, vol. 6, no. 3, pp. 1-13, 1996.

[10] C. Xinkai, "Design of a robust observer and its application for third order systems with disturbances," in Proc. IEEE Int. Conf. Syst., Man Cybern., Beijing, China, 1996, pp. 762-767.

[11] K. Erbatur, M. Okyay, and A. Sabanovic, "A study on robustness property of sliding-mode controllers: A novel design and experimental investigations," IEEE Trans. Ind. Electron., vol. 46, no. 5, pp. 1012-1018, Oct. 1999

[12] K. Erbatur and B. Calli, "Fuzzy boundary layer tuning as applied to the control of a direct drive robot," in Proc. IECON, 2007, pp. 2858-2863.

[13] Y. Salam and F. Wang, "Programmable analog VLSI neural circuits for control," in Proc. 28th IEEE Conf. Decis. Control, 1989, pp. 1765-1766.

[14] A. Stoica, R. Zebulum, D. Keymeulen, M. Ferguson, and V. Duong, "Fuzzy controller implementations with fewer than ten transistors?" in Proc. Congr. Evol. Comput., 2002, pp. 1830-1835.

[15] R. Zebulum, M. Vellasco, M. Pacheco, and H. Sinohara, "Evolvable hardware: On the automatic synthesis of analog control systems," in Proc. IEEE Aerosp. Conf., 2000, pp. 451-463.

[16] W. Perruquetti and J. Barbot, Sliding Mode Control in Engineering. New York: Marcel Dekker, 2002.

[17] V. Utkinn, Sliding Modes in Control and Optimization. New York: Springer-Verlag, 1992.

[18] H. Khalil, Nonlinear Systems. Englewood Cliffs, NJ: Prentice-Hall, 2002.

[19] KiNEDx Robot-User's Manual, Peak Robot., Colorado Springs, CO, 2007.

[20] S. Sanchez, A. Cabrera, I. Baturone, F. Moreno, and M. Brox, "FPGA implementation of embedded fuzzy controllers for robotic applications," IEEE Trans. Ind. Electron., vol. 54, no. 4, pp. 1937-1945, Aug. 2007.
[21] Y.-S. Kung, R.-F. Fung, and T.-Y. Tai, "Realization of a motion control IC for X-Y table based on novel FPGA technology," IEEE Trans. Ind. Electron., vol. 56, no. 1, pp. 43-53, Jan. 2009.

[22] G. Zhu, J. Penet, and L. Saydy, "Robust control of an electrostatically actuated MEMS in the presence of parasitics and parametric uncertainties," in Proc. Amer. Control Conf., Minneapolis, MN, 2006, pp. 1233-1238.

[23] A. Izadbakhsh and S. Rafiei, "Robust control methodologies for optical micro electro mechanical system-New approaches and comparison," in Proc. 13th Int. Power Electron. Motion Control Conf., 2008, pp. 2102-2107.

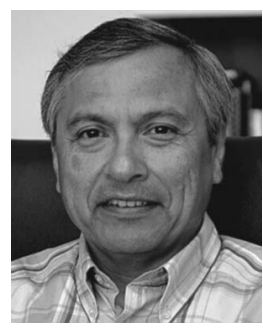

Joaquin Alvarez (M'92) was born in Tampico, Mexico, in 1953. He received the Diploma degree in electronics and telecommunications engineering from the School of Electrical and Mechanical Engineering, National Polytechnic Institute (NPI) Mexico City, Mexico, in 1975, and the Dr. Eng. degree in automatic control from the National School of Electrotechnical Engineering, National Polytechnique Institute of Grenoble, Saint Martin d'Heres, France, in 1979

He was a Professor with the Department of Electrical Engineering, Advanced Studies and Research Center, NPI. Since 1990, he has been a Full Professor with the Department of Electronics and Telecommunications, Scientific Research and Higher Education Center of Ensenada, Ensenada, Mexico. His current research interests are in the fields of nonlinear control, discontinuous systems, chaos control, and synchronization, with applications to mechanical systems and electronic circuits.

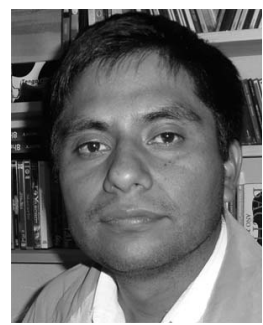

David Rosas was born in Tlaixpan, Mexico, in 1975 He received the B.S. degree in electronics engineering from Autonomous University of Puebla, Puebla, Mexico, in 1997, and the M.Sc. and Sc.D. degrees in electronics and telecommunications, with orientation in instrumentation and control, from Scientific Research and Higher Education Center of Ensenada, Ensenada, Mexico, in 1999 and 2005, respectively.

Since 1999, he has been a Full Professor with the Engineering Faculty, Autonomous University of Baja California, Mexicali, Mexico. His research interests are in the fields of nonlinear control, discontinuous systems, and synchronization.

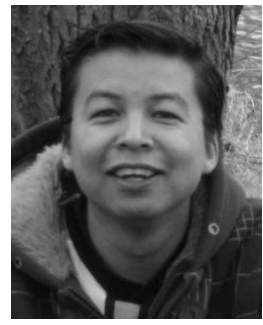

Jonatan Peña was born in Tulancingo, Mexico, on May 13, 1982. He received the degree in electronic engineering from the Universidad Autonoma del Estado de Hidalgo, Pachuca, Mexico, in 2005, and the M.Sc. degree in control and instrumentation from the Scientific Research and Higher Education Center of Ensenada (CICESE), Ensenada, Mexico, in 2007. $\mathrm{He}$ is currently working toward the Ph.D. degree in the Dynamics and Control Group, Department of Mechanical Engineering, Eindhoven University of Technology, Eindhoven, The Netherlands.

His research interests are in the areas of control and synchronization of mechanical systems, control of unmanned vehicles, sliding mode control, and nonlinear systems.

Mr. Peña's master thesis received the "Mejor tesis de Maestria" award from CICESE in 2008. 